PROCEEDINGS OF THE

AMERICAN MATHEMATICAL SOCIETY

Volume 125, Number 4, April 1997, Pages 987-999

S 0002-9939(97)03675-7

\title{
A FAMILY OF NEW UNIVERSAL $R$-MATRICES
}

\author{
KEQIN LIU
}

(Communicated by Roe Goodman)

\begin{abstract}
Left universal $R$-matrices and right universal $R$-matrices are introduced. A family of new universal $R$-matrices and charmed Hopf algebra is found.
\end{abstract}

\section{§1. INTRODUCTION}

In this paper, we will study a class of quasitriangular Hopf algebras $s \ell_{q, z}^{t}(2)$. Apart from the universal $R$-matrices, there are two other kinds of elements in $s \ell_{q, z}^{t}(2) \otimes s \ell_{q, z}^{t}(2)$, which are called left universal $R$-matrices and right universal $R$-matrices, respectively. Both left universal $R$-matrices and right universal $R$-matrices are solutions to the quantum Yang-Baxter equation. We find three varieties $V_{\ell}, V_{r}$ and $V$, which have the properties: each point in $V_{\ell}$ corresponds to a left universal $R$-matrix, each point in $V_{r}$ corresponds to a right universal $R$-matrix and each point in $V$ corresponds to a universal $R$-matrix. Therefore, we obtain not only a family of new solutions to the quantum Yang-Baxter equation parametrized by the points in the variety $V_{\ell} \cup V_{r}$, but also a family of new universal $R$-matrices parametrized by the points in the variety $V$. It is well known that the class of 3-manifold invariants introduced by N. Yu. Reshetikhin and V. G. Turaev are a linear combination of certain colored framed link invariants. The colored framed link invariants are special cases of the tangle operators for colored framed tangles. Therefore, the tangle operators play an important role in the study of 3-manifold invariants. As an application of the family of quasitriangular Hopf algebras, we will prove the existence of a class of tangle operators parametrized by the points in the variety $V$.

\section{§2. NeW UNIVERSAL $R$-MATRICES}

Let $H$ be a Hopf algebra with the comultiplication $\Delta$ and the antipode $S$. Let $\mathcal{R}:=\sum_{i} \alpha_{i} \otimes \beta_{i} \in H \otimes H, \mathcal{R}_{12}:=\sum_{i} x_{i} \otimes y_{i} \otimes 1, \mathcal{R}_{13}:=\sum_{i} x_{i} \otimes 1 \otimes y_{i}, \mathcal{R}_{23}:=$ $\sum_{i} 1 \otimes x_{i} \otimes y_{i}$ and $\Delta^{\prime}(x):=\sigma \Delta(x)$, where $\sigma$ is the twist automorphism of $H \otimes H$ given by $\sigma: x \otimes y \mapsto y \otimes x$ for $x, y \in H$. Consider the three conditions on $\mathcal{R}$ :

(a) $\mathcal{R} \Delta(x)=\Delta^{\prime}(x) \mathcal{R}$ for all $x \in H$,

(b) $(\Delta \otimes i d)(\mathcal{R})=\mathcal{R}_{13} \mathcal{R}_{23}$,

(c) $(i d \otimes \Delta)(\mathcal{R})=\mathcal{R}_{13} \mathcal{R}_{12}$.

Received by the editors May 25, 1994 and, in revised form, October 13, 1995.

1991 Mathematics Subject Classification. Primary 17B35, 17B37; Secondary 57Q45.

(C)1997 American Mathematical Society 
Definition 2.1. In the setting above, we have

1. $\mathcal{R}$ is called a left universal $R$-matrix if $\mathcal{R}$ satisfies (a) and (b).

2. $\mathcal{R}$ is called a right universal $R$-matrix if $\mathcal{R}$ satisfies (a) and (c).

3. $\mathcal{R}$ is called a universal $R$-matrix if $\mathcal{R}$ is invertible and satisfies (a), (b) and (c).

It is known that (a) and (b) imply the quantum Yang-Baxter equation:

$$
\mathcal{R}_{12} \mathcal{R}_{13} \mathcal{R}_{23}=\mathcal{R}_{23} \mathcal{R}_{13} \mathcal{R}_{12}
$$

Also, (a) and (c) imply the quantum Yang-Baxter equation. Hence, both a left universal $R$-matrix and a right universal $R$-matrix can be a solution to the quantum Yang-Baxter equation.

In the study of quantum groups, we have not seen any example of non-zero left (or right) universal $R$-matrices which is not a universal $R$-matrix. In order to give some such examples, we consider a Hopf algebra $s \ell_{q, z}(2)$, which is a generalization of the quantum group $s \ell_{q}(2)$. The Hopf algebra $s \ell_{q, z}(2)$ is defined as the associative algebra with generators $\left\{E, F, K^{ \pm 1}, z^{ \pm 1}\right\}$ and relations:

$$
\begin{aligned}
& K K^{-1}=K^{-1} K=1, \quad z z^{-1}=z^{-1} z=1 \text { and } z \text { is in the center of } s \ell_{q, z}^{t}(2), \\
& K E K^{-1}=q^{2} E, \quad K F K^{-1}=q^{-2} F, \quad E F-F E=\frac{K-z^{-1} K^{-1}}{q-q^{-1}}
\end{aligned}
$$

where the coproduct $\Delta$, the counit $\varepsilon$ and the antipode $S$ are defined by

$$
\begin{aligned}
& \Delta(E)=E \otimes 1+K \otimes E, \quad \varepsilon(E)=0, \quad S(E)=-K^{-1} E, \\
& \Delta(F)=F \otimes z^{-1} K^{-1}+1 \otimes F, \quad \varepsilon(F)=0, \quad S(F)=-F z K, \\
& \Delta(X)=X \otimes X, \quad \varepsilon(X)=1, \quad S(X)=X^{-1}
\end{aligned}
$$

for $X \in\left\{K^{ \pm 1}, z^{ \pm 1}\right\}$.

Throughout this paper, we assume that $q^{2}$ is a $t$-th primitive root of unity with $t \geq 2$. The ideal $\left\langle E^{t}, F^{t}, K^{t}-1, z^{t}-1\right\rangle$ of the algebra $s \ell_{q, z}(2)$ is a Hopf ideal of the Hopf algebra $s \ell_{q, z}(2)$. Set

$$
s \ell_{q, z}^{t}(2):=\frac{s \ell_{q, z}(2)}{\left\langle E^{t}, F^{t}, K^{t}-1, z^{t}-1\right\rangle} ;
$$

then we get a finite dimensional Hopf algebra $s \ell_{q, z}^{t}(2)$ with generators $\left\{E, F, K^{ \pm 1}\right.$, $\left.z^{ \pm 1}\right\}$ and relations:

$$
\begin{aligned}
& z z^{-1}=z^{-1} z=1 \text { and } z \text { is in the center of } s \ell_{q, z}^{t}(2), \\
& K K^{-1}=K^{-1} K=1, \\
& K E K^{-1}=q^{2} E, \quad K F K^{-1}=q^{-2} F \\
& E F-F E=\frac{K-z^{-1} K^{-1}}{q-q^{-1}} \\
& E^{t}=F^{t}=0, \quad K^{t}=z^{t}=1 .
\end{aligned}
$$


In order to describe a class of left (or right) universal $R$-matrices for $s \ell_{q, z}^{t}(2)$, we define three varieties $V_{\ell}, V_{r}$ and $V$ in $\mathbb{C}^{t^{2}}$ as follows:

$$
\begin{aligned}
V_{\ell} & :=\left\{\left(d_{\alpha \beta}\right)_{0 \leq \alpha, \beta<t} \mid \sum_{0 \leq \alpha<t} q^{2\left(\gamma^{\prime}-\gamma\right) \alpha} d_{\alpha, \gamma} d_{\beta-\alpha, \gamma^{\prime}}=\delta_{\gamma, \gamma^{\prime}} d_{\beta, \gamma}\right\}, \\
V_{r} & :=\left\{\left(d_{\alpha \beta}\right)_{0 \leq \alpha, \beta<t} \mid \sum_{0 \leq \alpha<t} q^{2\left(\beta^{\prime}-\beta\right) \alpha} d_{\beta, \alpha} d_{\beta^{\prime}, \gamma-\alpha}=\delta_{\beta, \beta^{\prime}} d_{\beta, \gamma}\right\}, \\
V & :=\left\{\left(d_{\alpha \beta}\right)_{0 \leq \alpha, \beta<t} \in V_{\ell} \cap V_{r} \mid \sum_{0 \leq \beta, \gamma<t} q^{-2 b \beta+2 d \gamma} d_{\beta \gamma} d_{-\beta-d, \gamma-b}=\delta_{(b, d),(0,0)}\right\},
\end{aligned}
$$

where $\gamma, \gamma^{\prime}, b, d \in\{0,1, \ldots, t-1\}$ and $d_{\alpha, \beta}=d_{\alpha^{\prime}, \beta^{\prime}}$ if $\alpha \equiv \alpha^{\prime} \bmod t$ and $\beta \equiv \beta^{\prime}$ $\bmod t$.

Note that the variety $V$ contains at least the following $t$ symmetric points:

$$
\left(x_{\alpha, \beta}\right)_{0 \leq \alpha, \beta<t}=\left(\begin{array}{cccc}
1 & 0 & \ldots & 0 \\
0 & 0 & \ldots & 0 \\
\vdots & \vdots & \ddots & \vdots \\
0 & 0 & \ldots & 0
\end{array}\right)
$$

and

$$
\left(x_{\alpha, \beta}\right)_{0 \leq \alpha, \beta<t}=\frac{1}{t}\left(\begin{array}{cccc}
1 & 1 & \ldots & 1 \\
1 & a & \ldots & a^{t-1} \\
\vdots & \vdots & \ddots & \vdots \\
1 & a^{t-1} & \ldots & a^{(t-1)^{2}}
\end{array}\right),
$$

where $a \in \mathbb{C}, a^{t}=1$ and $a \neq q^{2}$.

Example. If $t=2$, then

$$
\begin{aligned}
& V_{\ell}=\left\{\left(\frac{1}{2}, \frac{1}{2}, \frac{1}{2}, \frac{1}{2}\right),(1,0,0,0),(0,0,0,0)\right\} \cup\left\{(0,1,0,0),\left(0, \frac{1}{2}, 0, \pm \frac{1}{2}\right),\left(\frac{1}{2}, 0, \pm \frac{1}{2}, 0\right),\left(\frac{1}{2}, \frac{1}{2},-\frac{1}{2},-\frac{1}{2}\right)\right\} \\
& V_{r}=\left\{\left(\frac{1}{2}, \frac{1}{2}, \frac{1}{2}, \frac{1}{2}\right),(1,0,0,0),(0,0,0,0)\right\} \\
& \quad \cup\left\{(0,0,1,0),\left(0,0, \frac{1}{2}, \pm \frac{1}{2}\right),\left(\frac{1}{2}, \pm \frac{1}{2}, 0,0\right),\left(\frac{1}{2},-\frac{1}{2}, \frac{1}{2},-\frac{1}{2}\right)\right\} \\
& V=\left\{\left(\frac{1}{2}, \frac{1}{2}, \frac{1}{2}, \frac{1}{2}\right),(1,0,0,0)\right\} .
\end{aligned}
$$

Theorem 2.1. Let

$$
\begin{aligned}
& \mathcal{R}_{\left(d_{\alpha \beta}\right)_{0 \leq \alpha, \beta<t}} \\
& =\frac{1}{t} \sum_{0 \leq n, a, b, \gamma, \mu<t} \frac{\left(q^{-1}-q\right)^{n}}{[n] !} q^{2 a b+2 \mu \gamma-2 b \gamma+\frac{n(1-n)}{2}} d_{b-\mu, \gamma} E^{n} K^{a} z^{\gamma} \otimes F^{n} K^{b} z^{\mu},
\end{aligned}
$$

where $d_{\alpha, \beta} \in \mathbb{C}$. Then

(1) $\mathcal{R}_{\left(d_{\alpha \beta}\right)_{0 \leq \alpha, \beta<t}}$ is a left universal $R$-matrix for $s \ell_{q, z}^{t}(2)$ if and only if

$$
\left(d_{\alpha, \beta}\right)_{0 \leq \alpha, \beta<t} \in V_{\ell} .
$$

(2) $\mathcal{R}_{\left(d_{\alpha \beta}\right)_{0 \leq \alpha, \beta<t}}$ is a right universal $R$-matrix for $s \ell_{q, z}^{t}(2)$ if and only if

$$
\left(d_{\alpha, \beta}\right)_{0 \leq \alpha, \beta<t} \in V_{r} .
$$


(3) $\mathcal{R}_{\left(d_{\alpha \beta}\right)_{0 \leq \alpha, \beta<t}}$ is a universal $R$-matrix for $s \ell_{q, z}^{t}(2)$ if and only if

$$
\left(d_{\alpha, \beta}\right)_{0 \leq \alpha, \beta<t} \in V
$$

Proof. For convenience, we denote $\mathcal{R}_{\left(d_{\alpha \beta}\right)_{0 \leq \alpha, \beta<t}}$ by $\mathcal{R}$.

(1) It follows from a direct calculation that

$$
\mathcal{R} \Delta(x)=\Delta^{\prime}(x) \mathcal{R} \quad \text { for all } x \in s \ell_{q, z}^{t}(2)
$$

Hence, in order to prove (1), it suffices to prove

$$
(\Delta \otimes i d)(\mathcal{R})=\mathcal{R}_{13} \mathcal{R}_{23} \Longleftrightarrow\left(d_{\alpha, \beta}\right)_{0 \leq \alpha, \beta<t} \in V_{\ell}
$$

Set

$$
c_{n, a, b, \gamma, \mu}:=\frac{1}{t} \frac{\left(q^{-1}-q\right)^{n}}{[n] !} q^{2 a b+2 \mu \gamma-2 b \gamma+\frac{n(1-n)}{2}} d_{b-\mu, \gamma} .
$$

Recall that the $q$-analogue of the binomial theorem has the form:

$$
(A+B)^{n}=\sum_{k=0}^{n} q^{k^{2}-n k}\left(\begin{array}{l}
n \\
k
\end{array}\right) B^{k} A^{n-k}, \quad \text { where } B A=q^{2} A B .
$$

Let $A:=E \otimes 1$ and $B:=K \otimes E$; we get

$$
\Delta(E)^{n}=(E \otimes 1+K \otimes E)^{n}=\sum_{k=0}^{n} q^{k^{2}-n k}\left(\begin{array}{l}
n \\
k
\end{array}\right) K^{k} E^{n-k} \otimes E^{k}
$$

On one hand, using the fact above, we have

$$
\begin{aligned}
& (\Delta \otimes i d)(\mathcal{R})=(\Delta \otimes i d)\left(\sum_{0 \leq n, a, b, \gamma, \mu<t} c_{n a b \gamma \mu} E^{n} K^{a} z^{\gamma} \otimes F^{n} K^{b} z^{\mu}\right) \\
& =\sum_{0 \leq n, a, b, \gamma, \mu<t} c_{n a b \gamma \mu} \Delta(E)^{n} \Delta(K)^{a} \Delta(z)^{\gamma} \otimes F^{n} K^{b} z^{\mu} \\
& =\sum_{\substack{0 \leq n, a, b, \gamma, \mu<t \\
0 \leq k \leq n}} c_{n a b \gamma \mu} q^{k^{2}-n k}\left(\begin{array}{l}
n \\
k
\end{array}\right)\left(K^{k} E^{n-k} \otimes E^{k}\right)\left(K^{a} \otimes K^{a}\right)\left(z^{\gamma} \otimes z^{\gamma}\right) \otimes F^{n} K^{b} z^{\mu} \\
& =\sum_{\substack{0 \leq n, a, b, \gamma, \mu<t \\
0 \leq k \leq n}} c_{n a b \gamma \mu} q^{k(n-k)}\left(\begin{array}{l}
n \\
k
\end{array}\right) E^{n-k} K^{k+a} z^{\gamma} \otimes E^{k} K^{a} z^{\gamma} \otimes F^{n} K^{b} z^{\mu} .
\end{aligned}
$$


On the other hand, we have

$$
\begin{aligned}
& \mathcal{R}_{13} \mathcal{R}_{23}=\left(\sum_{0 \leq n^{\prime}, a^{\prime}, b^{\prime}, \gamma^{\prime}, \mu^{\prime}<t} c_{n^{\prime} a^{\prime} b^{\prime} \gamma^{\prime} \mu^{\prime}} E^{n^{\prime}} K^{a^{\prime}} z^{\gamma^{\prime}} \otimes 1 \otimes F^{n^{\prime}} K^{b^{\prime}} z^{\mu^{\prime}}\right) \\
& \times\left(\sum_{0 \leq n^{\prime \prime}, a^{\prime \prime}, b^{\prime \prime}, \gamma^{\prime \prime}, \mu^{\prime \prime}<t} c_{n^{\prime \prime} a^{\prime \prime} b^{\prime \prime} \gamma^{\prime \prime} \mu^{\prime \prime}} 1 \otimes E^{n^{\prime \prime}} K^{a^{\prime \prime}} z^{\gamma^{\prime \prime}} \otimes F^{n^{\prime \prime}} K^{b^{\prime \prime}} z^{\mu^{\prime \prime}}\right) \\
& =\sum_{\substack{0 \leq n^{\prime}, a^{\prime}, b^{\prime}, \gamma^{\prime}, \mu^{\prime}<t \\
0 \leq n^{\prime \prime}, a^{\prime \prime}, b^{\prime \prime}, \gamma^{\prime \prime}, \mu^{\prime \prime}<t}} c_{n^{\prime} a^{\prime} b^{\prime} \gamma^{\prime} \mu^{\prime}} c_{n^{\prime \prime} a^{\prime \prime} b^{\prime \prime} \gamma^{\prime \prime} \mu^{\prime \prime}} q^{-2 n^{\prime \prime} b^{\prime}} E^{n^{\prime}} K^{a^{\prime}} z^{\gamma^{\prime}} \otimes E^{n^{\prime \prime}} K^{a^{\prime \prime}} z^{\gamma^{\prime \prime}} \\
& \otimes F^{n^{\prime}+n^{\prime \prime}} K^{b^{\prime}+b^{\prime \prime}} z^{\mu^{\prime}+\mu^{\prime \prime}} \\
& =\sum_{\substack{0 \leq k \leq n<t \\
0 \leq a^{\prime}, b^{\prime}, \gamma^{\prime}, \mu^{\prime}<t \\
0 \leq a^{\prime \prime}, b, \gamma^{\prime \prime}, \mu<t}} c_{n-k, a^{\prime}, b^{\prime}, \gamma^{\prime}, \mu^{\prime}} c_{k, a^{\prime \prime}, b-b^{\prime}, \gamma^{\prime \prime}, \mu-\mu^{\prime}} q^{-2 k b^{\prime}} E^{n-k} K^{a^{\prime}} z^{\gamma^{\prime}} \otimes E^{k} K^{a^{\prime \prime}} z^{\gamma^{\prime \prime}} \\
& \otimes F^{n} K^{b} z^{\mu} \\
& =\sum_{\substack{a^{\prime} \equiv+a^{\prime \prime} \text { and } \\
\gamma^{\prime}=\gamma^{\prime \prime}}}+\sum_{\substack{a^{\prime} \not \equiv k+a_{\text {or }}^{\prime \prime} \bmod t \\
\gamma^{\prime} \neq \gamma^{\prime \prime}}}+
\end{aligned}
$$

Letting $a:=a^{\prime \prime}$ and $\gamma:=\gamma^{\prime}=\gamma^{\prime \prime}$ in the first sum, we get

$$
\begin{aligned}
& \mathcal{R}_{13} \mathcal{R}_{23} \\
& =\sum_{\substack{0 \leq k \leq n<t \\
0 \leq a, b, \gamma, \mu<t}}\left(\sum_{\substack{0 \leq b^{\prime}, \mu^{\prime}<t \\
0.2 k, k}} c_{n-k, k+a, b^{\prime}, \gamma, \mu^{\prime}} c_{k, a, b-b^{\prime}, \gamma, \mu-\mu^{\prime}} q^{-2 k b^{\prime}}\right) E^{n-k} K^{k+a} z^{\gamma} \\
& \otimes E^{k} K^{a} z^{\gamma} \otimes F^{n} K^{b} z^{\mu} \\
& +\sum_{\substack{0 \leq k \leq n<t \\
0 \leq a^{\prime}, \gamma^{\prime}, a^{\prime \prime}, b, \gamma^{\prime \prime}, \mu<t}}\left(\sum_{0 \leq b^{\prime}, \mu^{\prime}<t} c_{n-k, a^{\prime}, b^{\prime}, \gamma^{\prime}, \mu^{\prime}} c_{k, a^{\prime \prime}, b-b^{\prime}, \gamma^{\prime \prime}, \mu-\mu^{\prime}} q^{-2 k b^{\prime}}\right)
\end{aligned}
$$

$$
\times E^{n-k} K^{a^{\prime}} z^{\gamma^{\prime}} \otimes E^{k} K^{a^{\prime \prime}} z^{\gamma^{\prime \prime}} \otimes F^{n} K^{b} z^{\mu}
$$

Comparing $(2.2)$ with $(2.3)$, we see that $(\Delta \otimes i d)(\mathcal{R})=\mathcal{R}_{13} \mathcal{R}_{23}$ is equivalent to the following two equations:

$$
\begin{gathered}
\sum_{0 \leq b^{\prime}, \mu^{\prime}<t} c_{n-k, a^{\prime}, b^{\prime}, \gamma^{\prime}, \mu^{\prime}} c_{k, a^{\prime \prime}, b-b^{\prime}, \gamma^{\prime \prime}, \mu-\mu^{\prime}} q^{-2 k b^{\prime}}=0 \\
\text { for } a^{\prime} \not \equiv k+a^{\prime \prime} \quad \bmod t \text { or } \gamma^{\prime} \neq \gamma^{\prime \prime}, \\
\sum_{0 \leq b^{\prime}, \mu^{\prime}<t} c_{n-k, k+a, b^{\prime}, \gamma, \mu^{\prime}} c_{k, a, b-b^{\prime}, \gamma, \mu-\mu^{\prime}} q^{-2 k b^{\prime}}=c_{n, a, b, \gamma, \mu} q^{k(n-k)}\left(\begin{array}{l}
n \\
k
\end{array}\right) .
\end{gathered}
$$


Let $\alpha^{\prime}:=a^{\prime}-\gamma^{\prime}, \alpha^{\prime \prime}:=a^{\prime \prime}-\gamma^{\prime \prime}, \beta^{\prime}:=b^{\prime}-\mu^{\prime}$ and $\beta:=b-\mu$. Then the condition

$$
a^{\prime} \not \equiv k+a^{\prime \prime} \bmod t \quad \text { or } \quad \gamma^{\prime} \neq \gamma^{\prime \prime}
$$

is equavilent to the condition

$$
\alpha^{\prime} \not \equiv k+\alpha^{\prime \prime} \quad \bmod t \quad \text { or } \quad \gamma^{\prime} \neq \gamma^{\prime \prime},
$$

and by (2.1), we have

$$
\begin{aligned}
& \sum_{0 \leq b^{\prime}, \mu^{\prime}<t} c_{n-k, a^{\prime}, b^{\prime}, \gamma^{\prime}, \mu^{\prime}} c_{k, a^{\prime \prime}, b-b^{\prime}, \gamma^{\prime \prime}, \mu-\mu^{\prime}} q^{-2 k b^{\prime}} \\
= & \sum_{0 \leq \beta^{\prime}, \mu^{\prime}<t} c_{n-k, \alpha^{\prime}+\gamma^{\prime}, \beta^{\prime}+\mu^{\prime}, \gamma^{\prime}, \mu^{\prime}} c_{k, \alpha^{\prime \prime}+\gamma^{\prime \prime}, \beta^{\prime}+\mu^{\prime}-\beta^{\prime \prime}-\mu^{\prime \prime}, \gamma^{\prime \prime}, \mu-\mu^{\prime}} q^{-2 k\left(\beta^{\prime}+\mu^{\prime}\right)} \\
= & \frac{1}{t^{2}} \frac{\left(q^{-1}-q\right)^{n}}{[k] ![n-k] !} q^{\frac{n(1-n)}{2}+k(n-k)+2 \alpha^{\prime \prime} \mu+2 \gamma^{\prime \prime} \mu+2 \alpha^{\prime \prime} \beta} \\
& \quad \times\left(\sum_{0 \leq \beta^{\prime}<t} q^{2\left(\alpha^{\prime}-\alpha^{\prime \prime}-k\right) \beta^{\prime}} d_{\beta^{\prime} \gamma^{\prime}} d_{\beta-\beta^{\prime}, \gamma^{\prime \prime}}\right)\left(\sum_{0 \leq \mu^{\prime}<t} q^{\left.2\left(\alpha^{\prime}-\alpha^{\prime \prime}-k+\gamma^{\prime}-\gamma^{\prime \prime}\right) \mu^{\prime}\right)}\right),
\end{aligned}
$$

which is 0 if $\alpha^{\prime}-\alpha^{\prime \prime}-k+\gamma^{\prime}-\gamma^{\prime \prime} \not \equiv 0 \bmod t$, and which is

$$
\sum_{0 \leq \beta^{\prime}<t} q^{2\left(\gamma^{\prime \prime}-\gamma^{\prime}\right) \beta^{\prime}} d_{\beta^{\prime} \gamma^{\prime}} d_{\beta-\beta^{\prime}, \gamma^{\prime \prime}}
$$

if $\alpha^{\prime}-\alpha^{\prime \prime}-k+\gamma^{\prime}-\gamma^{\prime \prime} \equiv 0 \bmod t$. This means that

$$
(2.4) \Longleftrightarrow \sum_{0 \leq \beta^{\prime}<t} q^{2\left(\gamma^{\prime \prime}-\gamma^{\prime}\right) \beta^{\prime}} d_{\beta^{\prime} \gamma^{\prime}} d_{\beta-\beta^{\prime}, \gamma^{\prime \prime}}=0 \quad \text { for } \gamma^{\prime} \neq \gamma^{\prime \prime} \text {, }
$$

and

$$
(2.5) \Longleftrightarrow \sum_{0 \leq \beta^{\prime}<t} d_{\beta^{\prime} \gamma} d_{\beta-\beta^{\prime}, \gamma}=d_{\beta \gamma} .
$$

This proves (1).

(2) The proof is similar to the proof of (1).

(3) By Proposition 3.1 in [1],

$$
\mathcal{R} \text { is invertible } \Longleftrightarrow \mathcal{R}(S \otimes 1)(\mathcal{R})=1 \otimes 1=(S \otimes 1)(\mathcal{R}) \mathcal{R} .
$$

One can check that

$$
\begin{aligned}
\mathcal{R}(S \otimes 1)(\mathcal{R}) & =(S \otimes 1)(\mathcal{R}) \mathcal{R} \\
& =\sum_{0 \leq b, d<t} q^{-2 b d}\left(\sum_{0 \leq \beta, \gamma \leq t} q^{-2 b \beta+2 d \gamma} d_{\beta \gamma} d_{-\beta-d, \gamma-b}\right) z^{b} \otimes z^{d},
\end{aligned}
$$

which is $1 \otimes 1$ if and only if

$$
\sum_{0 \leq \beta, \gamma \leq t} q^{-2 b \beta+2 d \gamma} d_{\beta \gamma} d_{-\beta-d, \gamma-b}=\delta_{(b, d),(0,0)} .
$$

Hence, (3) is true. 


\section{$\S 3$. The ChARMED ELEMENTS $K^{-1} z^{\eta}$}

Let's recall the definition of charmed Hopf algebras from [3].

Definition 3.1. A Hopf algebra $H$ is called a quasitriangular Hopf algebra if there exists a universal $R$-matrix in $H \otimes H$, in which case we use $(H, \mathcal{R})$ to indicate that the quasitriangular Hopf algebra structure on $H$ is determined by the universal $R$-matrix $\mathcal{R}$.

Definition 3.2. Let $(H, \mathcal{R})$ be a quasitriangular Hopf algebra with the antipode $S$, and $\mathcal{R}=\sum_{i} \alpha_{i} \otimes \beta_{i} \in H \otimes H$. An invertible element $\mu$ in $H$ is called a charmed element associated with the universal $R$-matrix $\mathcal{R}$ if the following three conditions are satisfied:

(i) $\mu x \mu^{-1}=S^{2}(x)$ for all $x \in H$,

(ii) $\sum_{i} \alpha_{i} \mu^{-1} \beta_{i}=\sum_{i} \beta_{i} \mu \alpha_{i}$,

(iii) $S(\mu)=\mu^{-1}$.

A quasitriangular Hopf algebra $(H, \mathcal{R})$ is called a charmed Hopf algebra if $H$ contains a charmed element $\mu$, in which case we use $(H, \mathcal{R}, \mu)$ to indicate that the charmed Hopf algebra structure on $H$ is determined by the charmed element $\mu$ associated with the universal $R$-matrix $\mathcal{R}$.

According to Theorem 2.1, we have a family of quasitriangular Hopf algebras structures on $s \ell_{q, z}^{t}(2)$. When does the family of quasitriangular Hopf algebras contain charmed elements? Before answering this question, we need some identities.

For $m \in \mathbb{Z}$, we define

$$
\begin{gathered}
{[m]:=\frac{q^{m}-q^{-m}}{q-q^{-1}}, \quad[m] !:=[m][m-1] \ldots[1],} \\
\left(\begin{array}{c}
m \\
n
\end{array}\right):= \begin{cases}\frac{[m] !}{[n] ![m-n] !}, & \text { for } 1 \leq n<m, \\
1, & \text { for } n=0 \text { or } n=m,\end{cases} \\
{[m]_{q}:=\frac{q^{m}-1}{q-1}, \quad[m]_{q} !:=[m]_{q}[m-1]_{q} \ldots[1]_{q},} \\
\left(\begin{array}{l}
m \\
n
\end{array}\right)_{q}:= \begin{cases}\frac{[m]_{q} !}{[n]_{q} ![m-n]_{q} !}, & \text { for } 1 \leq n<m, \\
1, & \text { for } n=0 \text { or } n=m,\end{cases} \\
(K, z ; m]:=\frac{q^{m} K-q^{-m} z^{-1} K-1}{q-q^{-1}}, \\
\left(\begin{array}{c}
K, \quad m \\
j \quad
\end{array}\right):=\frac{[K, z ; m][K, z ; m-1] \ldots[K, z ; m-j+1]}{[j] !} .
\end{gathered}
$$

Lemma 3.1. In $s \ell_{q, z}^{t}(2)$, we have

$$
\begin{aligned}
& F^{n} E^{k}=\sum_{0 \leq i \leq \min (n, k)}(-1)^{i}\left(\begin{array}{c}
n \\
i
\end{array}\right)[i] !\left(\begin{array}{l}
k \\
i
\end{array}\right)[i] !\left(\begin{array}{ccc}
K, & z ; & n-k-1+i \\
i
\end{array}\right) E^{k-i} F^{n-i}, \\
& (3.1) \quad F^{n} E^{n}=\sum_{0 \leq j \leq i \leq n}(-1)^{i}\left(\begin{array}{c}
n \\
i
\end{array}\right)^{2}[i] ! \frac{q^{\frac{(2 j-i)(i-1)}{2}}}{\left(q-q^{-1}\right)^{i}}\left(\begin{array}{l}
i \\
j
\end{array}\right) E^{n-i} F^{n-i} K^{2 j-i} z^{j-i} .
\end{aligned}
$$


Proof. The proof is similar to the proof given in [3]. See [3] for details.

Now we present some interesting identities about $q$-integers.

Lemma 3.2. Let $q^{2}$ be a primitive $t$-th root of unity. Then

$$
\begin{gathered}
\sum_{0 \leq j \leq t-n-1-a} q^{-j(a+n+2)}\left(\begin{array}{c}
n+j+a \\
a+j
\end{array}\right)\left(\begin{array}{c}
a+j \\
j
\end{array}\right)=0 \\
\sum_{0 \leq j \leq i \leq t-n} q^{(2 p-2 j+i+2 n+1)(i-j)-(n+2) i}(-1)^{i+j}\left(\begin{array}{c}
n+i \\
i
\end{array}\right)\left(\begin{array}{c}
i \\
j
\end{array}\right)=q^{2(n+1)(1-p)},
\end{gathered}
$$

where $0 \leq n, p<t$ and $0 \leq a<t-n-1$.

Proof. Using the equality $\left(\begin{array}{l}n \\ k\end{array}\right)=q^{k^{2}-n k}\left(\begin{array}{l}n \\ k\end{array}\right)_{q^{2}}$, we have

or

$$
\begin{aligned}
X_{a}: & =\sum_{0 \leq j \leq t-n-1-a} q^{-j(a+n+2)}\left(\begin{array}{c}
n+j+a \\
a+j
\end{array}\right)\left(\begin{array}{c}
a+j \\
j
\end{array}\right) \\
& =\sum_{0 \leq j \leq t-n-1-a} q^{-j(a+n+2)} q^{(a+j)(-n)}\left(\begin{array}{c}
n+j+a \\
a+j
\end{array}\right)_{q^{2}} q^{j(-a)}\left(\begin{array}{c}
a+j \\
j
\end{array}\right)_{q^{2}},
\end{aligned}
$$

$$
\begin{aligned}
& q^{a n}[n]_{q^{2}} ![a]_{q^{2}} ! X_{a} \\
= & \sum_{0 \leq j \leq t-n-1-a} q^{-2 j(a+n+1)}[n+a+j]_{q^{2}}[n+a+j-1]_{q^{2}} \ldots[j+1]_{q^{2}} \\
= & \sum_{0 \leq j \leq t-1} q^{-2 j(a+n+1)}[n+a+j]_{q^{2}}[n+a+j-1]_{q^{2}} \ldots[j+1]_{q^{2}} \\
= & \frac{1}{\left(q^{2}-1\right)^{n+a}} \sum_{0 \leq j \leq t-1} q^{-2 j(a+n+1)}\left(\left(q^{2}\right)^{n+a+j}-1\right) \ldots\left(\left(q^{2}\right)^{j+1}-1\right) \\
= & \frac{1}{\left(q^{2}-1\right)^{n+a}} \sum_{0 \leq j \leq t-1} q^{-2 j(a+n+1)} \sum_{1 \leq k \leq 2^{n+a}}\left( \pm\left(q^{2}\right)^{f_{k} j+g_{k}}\right) \\
= & \frac{1}{\left(q^{2}-1\right)^{n+a}} \sum_{1 \leq k \leq 2^{n+a}}\left( \pm q^{2 g_{k}}\right) \sum_{0 \leq j \leq t-1}\left(q^{2}\right)^{\left(f_{k}-(a+n+1)\right) j},
\end{aligned}
$$

where $f_{k}$ and $g_{k}$ are some nonnegative integers for $1 \leq k \leq 2^{n+a}$.

Since $0 \leq f_{k} \leq n+a$ and $0 \leq a<t-n-1, f_{k}-(a+n+1) \not \equiv 0 \bmod t$ for all $1 \leq k \leq 2^{n+a}$. Therefore, we get

$$
\sum_{0 \leq j \leq t-1}\left(q^{2}\right)^{\left(f_{k}-(a+n+1)\right) j}=0 \quad \text { for all } 1 \leq k \leq 2^{n+a},
$$

which implies that $X_{a}=0$. This proves (3.2).

(3.3) follows from (3.2) directly.

We are ready to study when the family of quasitriangular Hopf algebras

$$
\left(s \ell_{q, z}^{t}(2), \mathcal{R}_{\left(d_{\alpha \beta}\right)_{0 \leq \alpha, \beta<t}}\right)
$$

contain charmed elements. Although we do not know if a charmed element is a group-like element, it is certain that group-like elements are good candidates for the charmed elements. 
For convenience, we set

$$
J:=z K
$$

Then the universal $R$-matrix $\mathcal{R}_{\left(d_{\alpha \beta}\right)_{0 \leq \alpha, \beta<t}}=\sum_{i} \alpha_{i} \otimes \beta_{i}$ in Theorem 2.1 can be reexpressed as

$$
\begin{aligned}
& \mathcal{R}_{\left(d_{\alpha \beta}\right)_{0 \leq \alpha, \beta<t}} \\
& \quad=\frac{1}{t} \sum_{0 \leq n, \alpha, \beta, \gamma, \mu<t} \frac{\left(q^{-1}-q\right)^{n}}{[n] !} q^{2 \alpha \beta+2 \alpha \mu+2 \gamma \mu+\frac{n(1-n)}{2}} d_{\beta \gamma} E^{n} K^{\alpha} J^{\gamma} \otimes F^{n} K^{\beta} J^{\mu} .
\end{aligned}
$$

Let us consider the group-like element $\mu=K^{\xi} J^{\eta}$ in $s \ell_{q, z}^{t}(2)$. It is easy to check that

$$
\begin{gathered}
S(\mu)=\mu^{-1} \\
S^{2}(x)=\mu x \mu^{-1} \quad \text { for } x \in s \ell_{q, z}^{t}(2) \text { iff } \xi+\eta \equiv-1 \bmod t .
\end{gathered}
$$

Now we begin to find the conditions on $\xi$ and $\eta$ which will imply the equation (ii) in Definition 3.2.

On one hand, we have

$$
\begin{aligned}
& \sum_{i} \alpha_{i} \mu^{-1} \beta_{i} \\
&= \frac{1}{t} \sum_{0 \leq n, \alpha, \beta, \gamma, \mu<t} \frac{\left(q^{-1}-q\right)^{n}}{[n] !} q^{2 \alpha \beta+2 \alpha \mu+2 \gamma \mu+\frac{n(1-n)}{2}} \\
& \times d_{\beta \gamma}\left(E^{n} K^{\alpha} J^{\gamma}\right)\left(K^{-\xi} J^{-\eta}\right)\left(F^{n} K^{\beta} J^{\mu}\right) \\
&= \frac{1}{t} \sum_{0 \leq n, \alpha, \beta, \gamma, \mu<t} \frac{\left(q^{-1}-q\right)^{n}}{[n] !} q^{2 \alpha \beta+2 \alpha \mu+2 \gamma \mu+\frac{n(1-n)}{2}+2 n(\xi-\alpha)+2 n(\eta-\gamma)} d_{\beta \gamma} \\
& \quad \times E^{n} F^{n} K^{\alpha+\beta-2 \xi} J^{\gamma+\mu-2 \eta} .
\end{aligned}
$$

Let $a:=\alpha+\beta-2 \xi$ and $b:=\gamma+\mu-2 \eta$. Then $\alpha=a-\beta+2 \xi$ and $\mu=b-\gamma+2 \eta$. So we get

$$
\begin{aligned}
& t\left(\sum_{i} \alpha_{i} \mu^{-1} \beta_{i}\right) K^{-\xi} J^{-\eta} \\
= & \sum_{0 \leq n, a, b<t} \frac{\left(q^{-1}-q\right)^{n}}{[n] !} q^{2(a+2 \xi)(b+2 \eta)+\frac{n(1-n)}{2}+2 n(\eta-a-\xi)} \\
& \times\left(\sum_{0 \leq \beta, \gamma<t} q^{2 \beta(a-\beta+2 \xi+\gamma-b-2 \eta)+2 \gamma(-a-2 \xi+b-\gamma+2 \eta)+2 n(\beta-\gamma)} d_{\beta \gamma}\right) E^{n} F^{n} K^{a} J^{b} \\
= & \sum_{0 \leq n, a, b<t} \frac{\left(q^{-1}-q\right)^{n}}{[n] !} q^{2(a+2 \xi)(b+2 \eta)+\frac{n(1-n)}{2}+2 n(\eta-a-\xi)} \\
& \times\left(\sum_{0 \leq \beta, \gamma<t} q^{2(a-b+n)(\beta-\gamma)-2 \beta^{2}+2 \beta \gamma-2 \gamma^{2}+4(\beta-\gamma)(\xi-\eta)} d_{\beta \gamma}\right) E^{n} F^{n} K^{a+b} z^{b} .
\end{aligned}
$$


Replacing $a$ by $p-b$, we have

$$
\begin{aligned}
& t\left(\sum_{i} \alpha_{i} \mu^{-1} \beta_{i}\right) K^{-\xi} J^{-\eta} \\
= & \sum_{0 \leq n, p, b<t} \frac{\left(q^{-1}-q\right)^{n}}{[n] !} q^{2(p-b+2 \xi)(b+2 \eta)+\frac{n(1-n)}{2}+2 n(\eta-p+b-\xi)} \\
& \quad \times\left(\sum_{0 \leq \beta, \gamma<t} q^{2(p-2 b+n)(\beta-\gamma)-2 \beta^{2}+2 \beta \gamma-2 \gamma^{2}+4(\beta-\gamma)(\xi-\eta)} d_{\beta \gamma}\right) E^{n} F^{n} K^{p} z^{b},
\end{aligned}
$$

or

$$
\begin{aligned}
& t\left(\sum_{i} \alpha_{i} \mu^{-1} \beta_{i}\right) K^{-\xi} J^{-\eta} \\
= & \sum_{0 \leq n, p, b<t} \frac{\left(q^{-1}-q\right)^{n}}{[n] !} q^{\frac{n(1-n)}{2}+2 n \eta+2 b(p+n-b)}\left(q^{2}\right)^{2 p \eta+4 \xi \eta-n p-n \xi+b(2 \xi-2 \eta)} \\
& \quad \times\left(\sum_{0 \leq \beta, \gamma<t} q^{2(p-2 b+n)(\beta-\gamma)-2 \beta^{2}+2 \beta \gamma-2 \gamma^{2}+4(\beta-\gamma)(\xi-\eta)} d_{\beta \gamma}\right) E^{n} F^{n} K^{p} z^{b},
\end{aligned}
$$

On the other hand, we have

$$
\begin{aligned}
& \sum_{i} \beta_{i} \mu \alpha_{i} \\
&= \frac{1}{t} \sum_{0 \leq n, \alpha, \beta, \gamma, \mu<t} \frac{\left(q^{-1}-q\right)^{n}}{[n] !} q^{2 \alpha \beta+2 \alpha \mu+2 \gamma \mu+\frac{n(1-n)}{2}} d_{\beta \gamma}\left(F^{n} K^{\beta} J^{\mu}\right)\left(K^{\xi} J^{\eta}\right)\left(E^{n} K^{\alpha} J^{\gamma}\right) \\
&= \frac{1}{t} \sum_{\substack{0 \leq n, \alpha, \beta, \gamma, \mu<t\\
}} \frac{\left(q^{-1}-q\right)^{n}}{[n] !} q^{2 \alpha \beta+2 \alpha \mu+2 \gamma \mu+\frac{n(1-n)}{2}+2 n(\xi+\beta)+2 n(\mu+\eta)} d_{\beta \gamma} \\
& \quad \times\left(F^{n} E^{n}\right) K^{\alpha+\beta+\xi} J^{\gamma+\mu+\eta} .
\end{aligned}
$$

By (3.1), we get

$$
\begin{aligned}
& t\left(\sum_{i} \beta_{i} \mu \alpha_{i}\right) K^{-\xi} J^{-\eta} \\
& =\sum_{\substack{0 \leq n, \alpha, \beta, \gamma, \mu<t \\
0 \leq j \leq i \leq n}} \frac{\left(q^{-1}-q\right)^{n}}{[n] !} q^{2 \alpha \beta+2 \alpha \mu+2 \gamma \mu+\frac{n(1-n)}{2}+2 n(\beta+\xi+\mu+\eta)} d_{\beta \gamma} \\
& \quad \times(-1)^{i}\left(\begin{array}{c}
n \\
i
\end{array}\right)^{2}[i] ! \frac{q^{\frac{(2 j-i)(i-1)}{2}}}{\left(q-q^{-1}\right)^{i}}\left(\begin{array}{c}
i \\
j
\end{array}\right) E^{n-i} F^{n-i} K^{\alpha+\beta+j} J^{\mu+\gamma+j-i} .
\end{aligned}
$$


Replacing $n$ by $n+i$, the equation above can be rewritten as

$$
\begin{aligned}
& t\left(\sum_{i} \beta_{i} \mu \alpha_{i}\right) K^{-\xi} J^{-\eta} \\
& =\sum_{\substack{0 \leq n, \alpha, \beta, \gamma, \mu<t \\
0 \leq j \leq i \leq t-n}} \frac{\left(q^{-1}-q\right)^{n}}{[n] !} \\
& \quad \times q^{2 \alpha \beta+2 \alpha \mu+2 \gamma \mu++2(n+i) \mu+\frac{n(1-n)}{2}+(i-1)(j-i)-n i+2(n+i)(\beta+\xi+\eta)} d_{\beta \gamma} \\
& \quad \times(-1)^{i+j}\left(\begin{array}{c}
n+i \\
i
\end{array}\right)\left(\begin{array}{c}
i \\
j
\end{array}\right) E^{n} F^{n} K^{\alpha+\beta+j} J^{\mu+\gamma+j-i} \\
& =\sum_{0 \leq n, a, b \leq t} \frac{\left(q^{-1}-q\right)^{n}}{[n] !} q^{2 a b+2 n b+2 n(\xi+\eta)+\frac{n(1-n)}{2}} \\
& \quad \times\left(\sum_{0 \leq \beta, \gamma<t} q^{2(a-b+n)(\beta-\gamma)-2 \beta^{2}+2 \beta \gamma-2 \gamma^{2}} d_{\beta \gamma}\right) \\
& \quad \times\left(\sum_{0 \leq j \leq i \leq t-n} q^{(2 a+2 b-2 j+i+2 n+1)(i-j)-n i+2 i(\xi+\eta)}(-1)^{i+j}\left(\begin{array}{c}
n+i \\
i
\end{array}\right)\left(\begin{array}{c}
i \\
j
\end{array}\right)\right) \\
& \quad \times E^{n} F^{n} K^{a} J^{b},
\end{aligned}
$$

or

$$
\begin{aligned}
& t\left(\sum_{i} \beta_{i} \mu \alpha_{i}\right) K^{-\xi} J^{-\eta} \\
& =\sum_{0 \leq n, p, b \leq t} \frac{\left(q^{-1}-q\right)^{n}}{[n] !} q^{\frac{n(1-n)}{2}+2 n \eta+2 b(p+n-b)}\left(q^{2}\right)^{n \xi} \\
& \quad \times\left(\sum_{0 \leq \beta, \gamma<t} q^{2(p-2 b+n)(\beta-\gamma)-2 \beta^{2}+2 \beta \gamma-2 \gamma^{2}} d_{\beta \gamma}\right) \\
& \quad \times\left(\sum_{0 \leq j \leq i \leq t-n} q^{(2 p-2 j+i+2 n+1)(i-j)-n i+2 i(\xi+\eta)}(-1)^{i+j}\left(\begin{array}{c}
n+i \\
i
\end{array}\right)\left(\begin{array}{c}
i \\
j
\end{array}\right)\right) \\
& \quad \times E^{n} F^{n} K^{p} z^{b} .
\end{aligned}
$$

Comparing (3.6) and (3.7), we see that

$$
\sum_{i} \alpha_{i} \mu^{-1} \beta_{i}=\sum_{i} \beta_{i} \mu \alpha_{i}
$$


if and only if $\xi$ and $\eta$ satisfy the following equation:

$$
\begin{aligned}
\left(q^{2}\right)^{2 p \eta+4 \xi \eta-n p-n \xi+b(2 \xi-2 \eta)} & \\
\times & \left(\sum_{0 \leq \beta, \gamma<t} q^{2(p-2 b+n)(\beta-\gamma)-2 \beta^{2}+2 \beta \gamma-2 \gamma^{2}+4(\beta-\gamma)(\xi-\eta)} d_{\beta \gamma}\right) \\
=\left(q^{2}\right)^{n \xi} & \left(\sum_{0 \leq \beta, \gamma<t} q^{2(p-2 b+n)(\beta-\gamma)-2 \beta^{2}+2 \beta \gamma-2 \gamma^{2}} d_{\beta \gamma}\right) \\
& \times\left(\sum_{0 \leq j \leq i \leq t-n} q^{(2 p-2 j+i+2 n+1)(i-j)-n i+2 i(\xi+\eta)}(-1)^{i+j}\left(\begin{array}{c}
n+i \\
i
\end{array}\right)\left(\begin{array}{c}
i \\
j
\end{array}\right)\right)
\end{aligned}
$$

Recall from (3.5) that $\xi+\eta \equiv-1 \bmod t$. Hence, the last bracket in the right hand side of (3.8) is equal to $q^{2(n+1)(1-p)}$ by (3.3). Replacing $\xi$ by $-\eta-1$ in (3.8), we get

$$
\begin{aligned}
& \left(q^{2}\right)^{(2 \eta+1)(p+n-2 b-(2 \eta+1))}\left(\sum_{0 \leq \beta, \gamma<t} q^{2(p-2 b+n)(\beta-\gamma)-2 \beta^{2}+2 \beta \gamma-2 \gamma^{2}+4(\beta-\gamma)(-2 \eta-1)} d_{\beta \gamma}\right) \\
& =\sum_{0 \leq \beta, \gamma<t} q^{2(p-2 b+n)(\beta-\gamma)-2 \beta^{2}+2 \beta \gamma-2 \gamma^{2}} d_{\beta \gamma},
\end{aligned}
$$

or

$$
\begin{aligned}
& \sum_{0 \leq \beta, \gamma<t} q^{2(p-2 b+n)(\beta-\gamma)-2 \beta^{2}+2 \beta \gamma-2 \gamma^{2}} \\
& \times\left(\left(q^{2}\right)^{(2 \eta+1)(p+n-2 b-(2 \eta+1)-2(\beta-\gamma))}-1\right) d_{\beta \gamma}=0
\end{aligned}
$$

for all $0 \leq n, p, b<t$.

In summary, we have obtained the main theorem:

Theorem 3.1. Let $\mathcal{R}_{\left(d_{\alpha \beta}\right)_{0 \leq \alpha, \beta<t}}$ be the universal $R$-matrix in Theorem 2.1, where $\left(d_{\alpha \beta}\right)_{0 \leq \alpha, \beta<t}$ is a point in the variety $V$. Then the group-like element $K^{\xi} z^{\eta}$ is a charmed element in the quasitriangular Hopf algebra $\left(s \ell_{q, z}^{t}(2), \mathcal{R}_{\left(d_{\alpha \beta}\right)_{0 \leq \alpha, \beta<t}}\right)$ if and only if $\xi=-1$ and $\eta$ satisfies the equation (3.9).

As a corollary, we have the following theorem.

Theorem 3.2. If $t$ is odd and $\left(d_{\alpha \beta}\right)_{0 \leq \alpha, \beta<t} \in V$, then $\left(s \ell_{q, z}^{t}(2), \mathcal{R}_{\left(d_{\alpha \beta}\right)_{0 \leq \alpha, \beta<t}}\right)$ is a charmed Hopf algebra with the charmed element $K^{-1} z^{\frac{t-1}{2}}$.

Proof. If $\eta=\frac{t-1}{2}$, then $\left(q^{2}\right)^{2 \eta+1}=1$. Hence, $(3.9)$ is true for all points $\left(d_{\alpha \beta}\right)_{0 \leq \alpha, \beta<t}$ $\in V$. It follows from Theorem 3.1 that Theorem 3.2 holds.

R. Kirby and P. Melvin in [3] proved that every charmed Hopf algebra yields a tangle operator. Combining Theorem 3.6 in [3] and Theorem 3.2 above, we get

Theorem 3.3. For every odd $t$ and every point $\left(d_{\alpha \beta}\right)_{0 \leq \alpha, \beta<t} \in V$, there is a tangle operator $\mathcal{F}_{t ;\left(d_{\alpha \beta}\right)_{0 \leq \alpha, \beta<t}}$ for colored framed tangles which behaves well with respect to compositions and tensor products. 
Remark. We call $s \ell_{q, z}(2)$ the centralized quantum group for $s \ell(2)$. Similarly, there are the centralized quantum groups $U_{q, z_{1}, z_{2}, \ldots, z_{n}}(\mathcal{G})$ for $\mathcal{G}$ a finite-dimensional semisimple Lie algebra of rank $n$ over $\mathbb{C}$. We have found that Lusztig's braid group action on the Drinfeld-Jimbo quantum groups $U_{q}(\mathcal{G})$ can be generalized to the centralized quantum groups $U_{q, z_{1}, z_{2}, \ldots, z_{n}}(\mathcal{G})$.

\section{ACKNOWLEDGEMENT}

I thank Prof. J. B. Carrell and NSERC (Canada) for support. I thank Prof. R.V. Moody and Prof. D. Rolfsen for useful conversations. I thank the referee for his helpful comments.

\section{REFERENCES}

1. V. G. Drinfeld, On almost cocommutative Hopf algebras, Leninggrad Math. J. 1,No.2 (1990), 321-342. MR 91b:16046

2. M. Jimbo, A q-difference analogue of $U(\mathfrak{G})$ and the Yang-Baxter equation, Lett. Math. Phys. 10 (1985), 63-69. MR 86k:17008

3. R. Kirby and P. Melvin, The 3-manifold invariants of Witten and Reshetikhin-Turaev for $s \ell(2, \mathbb{C})$, Inv. Math. 105 (1991), 473-545. MR 92e:57011

4. M. Okado and H. Yamane, R-matrices with gauge parameters and multi-parameter quantized enveloping algebras, Special Functions (Okayama, 1990), ICM 90 Satellite Conf. Proc., Springer, Tokyo, 1991, pp. 289-293. MR 93f:17025

5. N. Reshetikhin and V.G.Turaev, Ribbon graphs and their invariants derived from quantum groups, Commun. Math. Phys. 127 (1990), 1-26. MR 91c:57016

6. N. Reshetikhin and V.G.Turaev, Invariants of 3-manifolds via link polynomials and quantum groups, Inv. Math. 103 (1991), 547-597. MR 92b:57024

7. M. E. Sweedler, Hopf Algebras, Math. Lecture Notes Series, Benjamin, New York, 1969. MR 40:5705

Department of Mathematics, The University of British Columbia, Vancouver, British Columbia, Canada V6T 1Z2

E-mail address: kliu@math.ubc.ca 